\title{
Dynamics of morphological evolution in experimental Escherichia coli populations
}

\author{
F. Cui and B. Yuan \\ Department of Computer Science and Engineering, \\ Shanghai Jiao Tong University, Shanghai, China \\ Corresponding authors: F. Cui / B. Yuan \\ E-mail: cuifangshu001@163.com / boyuan@sjtu.edu.cn
}

Genet. Mol. Res. 15 (3): gmr.15038991

Received July 21, 2016

Accepted August 1, 2016

Published August 30, 2016

DOI http://dx.doi.org/10.4238/gmr.15038991

Copyright (C) 2016 The Authors. This is an open-access article distributed under the terms of the Creative Commons Attribution ShareAlike (CC BY-SA) 4.0 License.

\begin{abstract}
Here, we applied a two-stage clonal expansion model of morphological (cell-size) evolution to a long-term evolution experiment with Escherichia coli. Using this model, we derived the incidence function of the appearance of cell-size stability, the waiting time until this morphological stability, and the conditional and unconditional probabilities of morphological stability. After assessing the parameter values, we verified that the calculated waiting time was consistent with the experimental results, demonstrating the effectiveness of the twostage model. According to the relative contributions of parameters to the incidence function and the waiting time, cell-size evolution is largely determined by the promotion rate, i.e., the clonal expansion rate of selectively advantageous organisms. This rate plays a prominent role in the evolution of cell size in experimental populations, whereas all other evolutionary forces were found to be less influential.
\end{abstract}

Key words: Two-stage clonal expansion model; Morphological evolution; Morphological stability; Evolution experiment; Escherichia coli 


\section{INTRODUCTION}

A central aim of evolutionary biology is a deeper understanding of the mechanisms of morphological evolution. Despite considerable progress towards this end, much research is still devoted to the study of morphological evolution. For these investigations, evolutionary biologists resort to fossils and examination of natural populations (Jablonski, 2000; Huey et al., 2000; Thomas et al., 2001; Márquez-García et al., 2009; Futuyma, 2010; Mahler et al., 2010; Matamorovidal et al., 2012). However, a more explicit and complete understanding remains elusive due to difficulties in the application of the fossil data to the natural population. Therefore, some biologists have started to conduct evolution experiments on various organisms in order to explore issues pertaining to morphological evolution. In 1988, Lenski et al. used Escherichia coli to perform a long-term laboratory evolution experiment. E. coli exhibits many features that are advantageous for evolution experiments. For instance, it has a short generation time and large population size, and it can be kept in suspended animation and later resuscitated, allowing for direct comparison of evolutionary change in ancestral and evolved types. Because many variables can be controlled, this laboratory evolution experiment provides the opportunity to rigorously explore questions pertaining to morphological evolution. The materials and methods that involve the ancestral strain, culture conditions, means for checking contamination, measurement of average cell volume, etc. are demonstrated in the literature (Lenski et al., 1991; Elena and Lenski, 2003; Lenski, 2004). This type of evolution experiment has illustrated more extensive and complicated evolution kinetics than theoretical studies on single populations or alleles. In this long-term experiment, we detected that morphology (cell size) increased quickly in all 12 of the experimental populations for the first 2000 generations in the unchanged experimental condition. However, cell size was ultimately nearly static, which is morphological stasis (or near stasis) (Lenski and Travisano, 1994).

In that evolution experiment, mutations in the $p b p A$ operon were shown to be responsible for cell-size evolution in two populations (Philippe et al., 2009). There are also mutations in the pbpA operon in four other populations, which likely affect the changes in cell volume (Woods et al., 2006). Additionally, only one of the 12 populations has a mutation that is upstream of the glmUS operon, which contributes to adaptation, involving the evolution in cell size (Stanek et al., 2009). However, due to the increment in the average cell size in all 12 populations, and the limited influence of the above mutations on the increase in cell volume, it is possible that other mutations could have an effect on evolved changes in cell size. Moreover, the selective advantage or other evolutionary reasons for changes in cell volume remain unintelligible.

In an effort to fully understand cell-size evolution, we resort to the application of mathematical models. Armitage and Doll (1954) and Knudson (1971) used mathematical models to describe the evolutionary process and investigate the anticipated time to achieve a certain number of mutations. Iwasa et al. (2004) analyzed a two-stage model for depicting a cell population evolving in terms of the Moran model. Beerenwinkel et al. (2007) examined the waiting time until the occurrence of several mutations according to the Wright-Fisher model when the population size is large. Schweinsberg (2008) and Durrett et al. (2009) developed asymptotic distributions for the anticipated time to a number of mutations. Gerstung and Beerenwinkel (2010) described the accumulation of mutations using conjunctive Bayesian networks. Moreover, there is a mathematical model that connects cell physiology with population genetics to replicate parallel increases in cell size and fitness observed in the evolution experiment with E. coli (Graña and Acerenza, 2001). In this study, we developed

Genetics and Molecular Research 15 (3): gmr.15038991 
a two-stage clonal expansion model (Moolgavkar, 1978; Moolgavkar and Venzon, 1979; Moolgavkar and Knudson, 1981) to describe morphological evolution in an E. coli evolution experiment in a constant environment. We derived the incidence function of emergence of cell-size stability, calculated the waiting time until this morphological stability, and expressed the conditional and unconditional probabilities. Furthermore, with the aim of analyzing and interpreting this morphological (cell-size) evolution under various evolutionary forces, we determined how the various parameters affect the incidence, function, and waiting time. Finally, we compared the dynamics of cell-size evolution obtained by the two-stage clonal expansion model and the Wright-Fisher model.

\section{MATERIAL AND METHODS}

We assume that two rate-limiting events are required to reach morphological (cellsize) stability after the introduction of $E$. coli into the constant experimental environment. In other words, the genetic progress of cell size evolves on the basis of a two-stage clonal expansion model. In the long-term experiment, there are 12 replicate E. coli populations (Lenski et al., 1991). They have the same ancestor, and have been procreated in an identical environment since 1988. Therefore, each population has no initial genetic variance, and completely depends on new mutations in the following evolution. We consider each population of $E$. coli to be wild type at time (generation) $t=0$, with a constant population size $N$. These wild-type organisms (W-organisms) mutate to organisms of an intermediate shape (I-organisms) with a stochastic event rate of $\mu_{1}(t)$. These I-organisms can split into two groups of I-organisms at a stochastic rate $\alpha(t)$ and can die or differentiate at rate $\beta(t)$. Additionally, I-organisms can split into an I-organism and a morphologically stable organism (S-organism) at a second stochastic event rate of $\mu_{2}(t)$ (Figure 1). In the experimental population of $E$. coli, the cell volume eventually becomes static (or near static), demonstrating that the organisms have run out of ways to increase their cell size and continue to adapt to the unaltered environment. Within this two-stage model, the formation of a single S-organism is presumed to result in the eventual stasis of cell size in the longterm experiment. The lag time that occurs between the generation of a single S-organism and the generation of a morphologically stable population can be neglected.

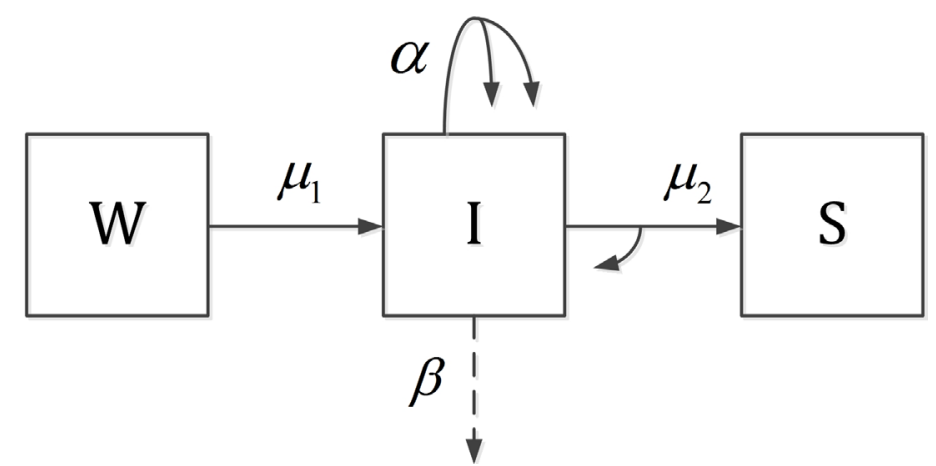

Figure 1. Schematic representation of the two-stage clonal expansion model. W, wild-type organisms (W-organisms); I, intermediate organisms (I-organisms); S, morphologically stable organisms (S-organisms).

Genetics and Molecular Research 15 (3): gmr.15038991 


\section{Incidence function}

In the two-stage model of clonal growth, the most essential quantity is the incidence function at time (generation) $t$, denoted by $h(t)$, which is the instantaneous rate of emergence of the first S-organism in a population. With time, the incidence function $h(t)$ increases first linearly and then exponentially to reach a constant asymptotic value. This is also observed at $t=0$ and $h(0)=0$. However, we can only observe the linear growth of the incidence function with numerous data sets. Therefore, it is sufficient to use a simplified form of the two-stage model in this case (Chen, 1993; Heidenreich, 1996).

Initially, at time $t=0$, all members of the population of $N$ individuals of $E$. coli are $\mathrm{W}$-organisms. The expected number of I-organisms, $y(t)$, is given by the ordinary differential equation (ODE)

$$
d y(t) / d t=N \mu_{1}(t)+[\alpha(t)-\beta(t)] y(t) \quad y(0)=0 \quad \text { (Equation 1) }
$$

The rate of appearance of the first S-organism in the population can be written as:

$$
h(t)=\mu_{2}(t) y(t)
$$

(Equation 2)

In the long-term evolution experiment, there are no mechanisms for genetic exchange and no artificial special traits of organisms after the $E$. coli is introduced into the constant environment. Therefore, the parameters $\mu_{1}, \alpha, \beta$, and $\mu_{2}$ should be constant. Therefore, we can obtain the solution of Equation 1 as follows:

$$
y(t)=\frac{N \mu_{1}}{\alpha-\beta}\{\exp [(\alpha-\beta) t]-1\}
$$

(Equation 3)

Accordingly, the incidence function is of the form:

$$
h(t)=\frac{N \mu_{1} \mu_{2}}{\alpha-\beta}\{\exp [(\alpha-\beta) t]-1\}
$$

(Equation 4)

\section{Waiting time to morphological stability}

The survival function for this simplified two-stage model is defined as

$$
S(t)=\exp [-H(t)]
$$

where $H(t)$ is the integrated incidence

$$
H(t)=\int_{0}^{t} h(\tilde{t}) d \tilde{t}=\frac{N \mu_{1} \mu_{2}}{(\alpha-\beta)^{2}}\{\exp [(\alpha-\beta) t]-(\alpha-\beta) t-1\} \quad \text { (Equation 6) }
$$

Genetics and Molecular Research 15 (3): gmr.15038991 
Within this model, the waiting time to cell-size stability in the evolution experiment can be estimated as the median waiting time $T$ to the first S-organism, which satisfies the equation $S(T)=1 / 2$, i.e. $H(T)=\ln 2$. The solution of this equation is

$$
T=\frac{-A-W_{-1}\left(-e^{-A}\right)}{\alpha-\beta}
$$

where $A=\frac{(\alpha-\beta)^{2} \ln 2}{N \mu_{1} \mu_{2}}+1$, and $W_{-1}$ is the branch of the Lambert $\mathrm{W}$ function that is indexed by -1 . The Lambert $\mathrm{W}$ function is the inverse function of $f(x)=x e^{x}$ (Corless et al., 1996). Moreover, $W_{-1}$ can be expanded into series as follows (Schöllnberger et al., 2010):

$$
W_{-1}(x)=\ln (-x)-\ln [-\ln (-x)]+O\left(\frac{\ln [-\ln (-x)]}{\ln (-x)}\right)
$$

Consequently, the median waiting time until the first S-organism appears can be approximated as:

$$
T \approx \frac{\ln A}{\alpha-\beta}=\frac{\ln \left[\frac{(\alpha-\beta)^{2} \ln 2}{N \mu_{1} \mu_{2}}+1\right]}{\alpha-\beta}
$$

\section{Probability of morphological stability}

According to the two-stage clonal growth model, the probability-generating function $\Psi$ for the generation of I-organisms and S-organisms out of $N \mathrm{~W}$-organisms can be deduced and is dependent on the probability-generating function $\Phi$ for the generation of I-organisms out of initially one I-organism and zero S-organisms (Hoogenveen et al., 1999). When the number of I-organisms, $y$, and the number of S-organisms, $z$, are time-constant parameters, the probability-generating function $\Phi$ can be solved:

$$
\Phi(y, z ; t)=B(z)+\frac{C(z)-B(z)}{1-\frac{y-C(z)}{y-B(z)} \exp (\alpha[C(z)-B(z)] t)}
$$

Here, $0<B(z)<1$ and $C(z) \geq 1$, indicate the two roots of the characteristic equation:

$$
\alpha x^{2}-\left[\alpha+\beta+(1-z) \mu_{2}\right] x+\beta=0
$$

(Equation 11)

Then, the probability generating function $\Psi$ is given by:

$$
\begin{aligned}
& \Psi(y, z ; t)=\exp \left(\int_{v \leq t} \mu_{1} N[\Phi(y, z ; v)-1] d v\right) \\
& =\exp \left(\frac{\mu_{1} N}{\alpha}\{\alpha(C-1) t+\ln (C-B)-\ln [y-B+(C-y) \exp (\alpha(C-B) t)]\}\right)
\end{aligned}
$$

The conditional probability that there are zero S-organisms at $t$ starting from one I-organism, $P(S(t)=0 \mid I(0)=1)$, can be defined by $\Phi(1,0 ; t)$. Then, substituting $z=0$ in Equation (11), we obtain the two roots, which can be approximated as $B \approx \beta / \alpha$ and $\mathrm{C} \approx 1+\mu_{2} / \alpha$. Therefore, the conditional probability is:

Genetics and Molecular Research 15 (3): gmr.15038991 


$$
P(S(t)=0 \mid I(0)=1)=\frac{\beta}{\alpha}+\frac{1+\mu_{2} / \alpha-\beta / \alpha}{1+\frac{\mu_{2} / \alpha}{1-\beta / \alpha} \exp \left[\left(\alpha+\mu_{2}-\beta\right) t\right]}
$$

In addition, the unconditional probability of zero S-organisms at $t$ in the experimental population is:

$$
\begin{aligned}
& P(S(t)=0)=\Psi(1,0 ; t) \\
& =\exp \left(\frac{\mu_{1} N}{\alpha}\left\{\mu_{2} t+\ln \left(1+\mu_{2} / \alpha-\beta / \alpha\right)-\ln \left[1-\beta / \alpha+\frac{\mu_{2}}{\alpha} \exp \left(\left(\alpha+\mu_{2}-\beta\right) t\right)\right]\right\}\right)
\end{aligned}
$$

Furthermore, the conditional probability of one S-organism at $t$ in one experimental population, given that there is initially one I-organism, can be written as:

$$
P(S(t)=1 \mid I(0)=1)=\frac{\alpha-\beta}{\alpha}-\frac{1+\mu_{2} / \alpha-\beta / \alpha}{1+\frac{\mu_{2} / \alpha}{1-\beta / \alpha} \exp \left[\left(\alpha+\mu_{2}-\beta\right) t\right]}
$$

The unconditional probability that there is one S-organism at $t$ is given by:

$$
P(S(t)=1)=\exp \left(\frac{\mu_{1} N}{\alpha}\{\ln (1-\beta / \alpha)-\ln [-\beta / \alpha+\exp ((\alpha-\beta) t)]\}\right)
$$

\section{RESULTS}

We used a two-stage growth model to investigate cell-size dynamics in evolving populations of $E$. coli. Using this model, we expressed the incidence function of emergence of cell-size stability in a long-term experiment and computed the waiting time to morphological stability, which is an analytical approximation from the median waiting time $T$ to the emergence of the first S-organism. Moreover, we also provide the conditional and unconditional probabilities for the generation of S-organisms. We used the following parameter values in this two-stage model: the first event rate $\mu_{1}=1 \times 10^{-10}$ per generation, the promotion rate (the growth rate of I-organisms) $\alpha-\beta=0.005$ per generation, the second event rate $\mu_{2}=1 \times 10^{-8}$ per generation, and the population size $N=5 \times 10^{8}$ (Lenski and Travisano, 1994; Barrick et al., 2009).

As shown in Figure 2, the incidence function is powerfully affected by the promotion rate $\alpha-\beta$, whereas the first event rate $\mu_{1}$ has less influence. Similar to $\mu_{1}$, the second event rate $\mu_{2}$ and the population size $N$ enter linearly into the incidence function, and thus also exert a weaker effect. Accordingly, the promotion rate is the dominant force in the evolutionary dynamics of cell size in experimental populations in a constant environment.

Using these parameter values, we evaluated the median waiting time $T$, which correlates with the results of the long-term evolution experiment (Figure 3 ). Therefore, this two-step model is a good fit for the genetic progress of cell size. To explore the dependence of the median waiting time $T$ on the parameters $\mu_{1}, \alpha-\beta, \mu_{2}$, and $N$, we plotted $T$ against varying parameter values of the first event rate $\mu_{1}$ ranging from $1 \times 10^{-11}$ to $1 \times 10^{-8}$, values of the promotion rate $\alpha-\beta$ ranging from $5 \times 10^{-4}$ to $5 \times 10^{-1}$, values of the second event rate $\mu_{2}$ ranging from $1 \times 10^{-9}$ to $1 \times 10^{-6}$, and values of the population size $N$ ranging from $5 \times 10^{6}$ to 5 $\mathrm{x} 10^{9}$ (Figure 3 ). When time $T$ is plotted against one parameter, the other parameter values are set as given above. As a result, Figure 3 shows that the median waiting time $T$ decreases with

Genetics and Molecular Research 15 (3): gmr.15038991 
increasing $\mu_{1}, \alpha-\beta, \mu_{2}$, and $N$. Therefore, $T$ exhibits the strongest dependency on the growth rate $\alpha-\beta$, confirming a major role of the promotion rate in the process of cell-size evolution in the long-term experiment. Because of the difficulty in obtaining parameter values for $\alpha$ and $\beta$, we only derived analytic expressions for conditional and unconditional probabilities for the generation of S-organisms in the two-stage growth model of cell-size evolution.
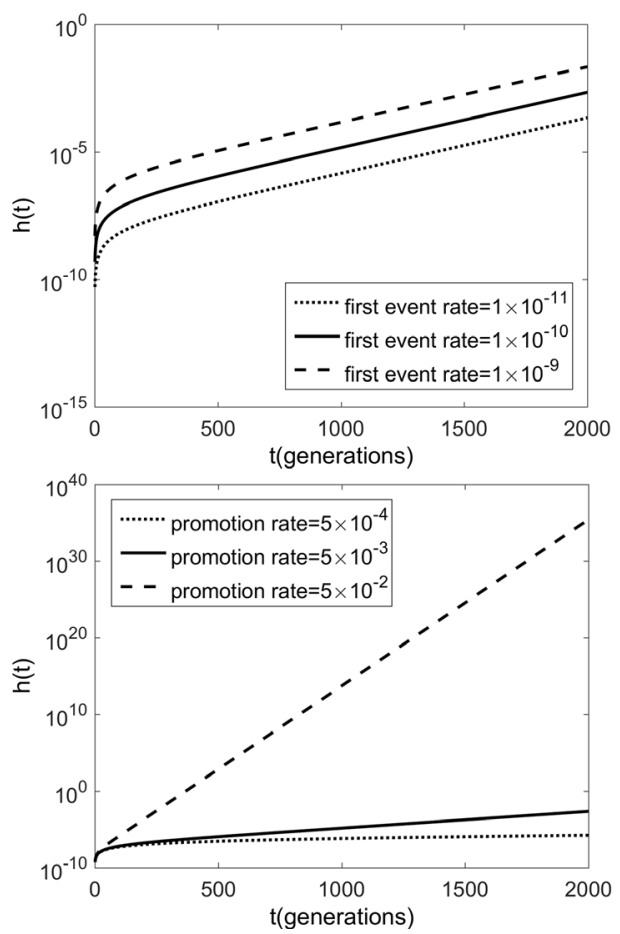

Figure 2. The incidence function $h(t)$ is plotted versus the time $t$ for three different first event rates (above) and three different promotion rates (below). Parameter values include $\mu_{2}=1 \times 10^{-8}$ per generation for the second event rate and $N=5 \times 10^{8}$ for the population size.

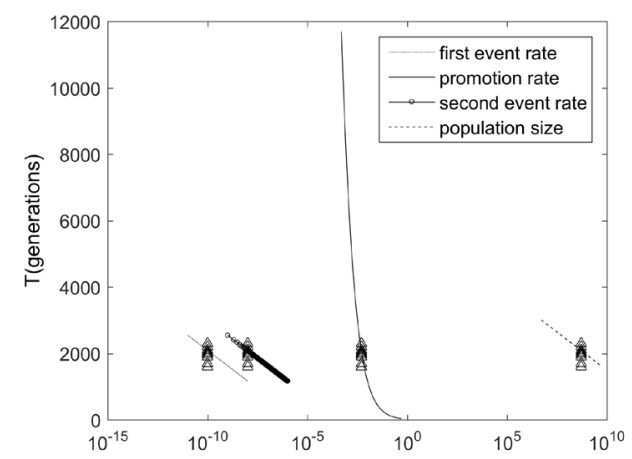

Figure 3. The median waiting time $T$ to the first S-organism as a function of the first event rate $\mu_{1}$, the promotion rate $\alpha-\beta$ (the growth rate of I-organisms), the second event rate $\mu_{2}$, and the population size $N$. Triangles represent experimental data from the long-term $E$. coli evolution experiment.

Genetics and Molecular Research 15 (3): gmr.15038991 


\section{DISCUSSION}

In addition to the above model, we employed a population genetic model, the Wright-Fisher model, to investigate the genetic progress of cell size in the evolution experiment (Cui and Yuan, 2012; Cui and Yuan, 2015). In the Wright-Fisher model, cellsize evolution is described as an accumulation of mutations with selective advantage in susceptible genes involved in cell size. In contrast, the two-stage clonal expansion model regards this evolutionary process as the end result of two crucial, specific, irreversible, rate-restrictive, and hereditary genomic events. The two-event rates, $\mu_{1}$ and $\mu_{2}$, do not represent genomic mutation rates, but rather conversion rates between the stages of morphological evolution. For calculation of the waiting time to final stasis (near stasis) in cell size in the Wright-Fisher model, we compute this waiting time as the time until the emergence of an organism with $m$ mutations in the evolving population of $E$. coli. By comparison, in the two-step model, this waiting time is estimated by the median time to the occurrence of the first S-organism.

The two different models have conceptual affinities, as both interpret cell-size stability as occurring via cumulative mutations with selective advantage and clonal expansion of selectively favorable organisms. Moreover, the growth rate, $\alpha-\beta$, of I-organisms in the two-stage model plays an analogous role to the average selective advantage, $s$, in the Wright-Fisher model. Therefore, the two parameters $s$ and $\alpha-\beta$ can be regarded as the clonal growth rate of selectively advantageous organisms and thus represent selection parameters.

In the Wright-Fisher model of cell-size evolution, we calculated the expected waiting time to reach cell-size stability as:

$$
t_{m} \approx m\left[\ln \left(1+s \sqrt{\ln \left(N^{2} /(2 \pi)\right)} /(u l)\right)\right]^{2} /\left[s \ln \left(N^{2} /(2 \pi)\right)\right]
$$

(see equation (19) in Cui and Yuan, 2015). By comparing this calculation with the equation for waiting time to cell-size stability in the two-stage clonal expansion model (see Equation (9)), we find that the two equations are obviously similar. In both expressions, the selection parameters $s$ and $\alpha-\beta$ appear in the denominator, whereas all other parameters appear only in the logarithm. Accordingly, the two models give the same conclusion - namely, that the selection parameter has the most powerful effect on waiting time, implying that cell-size evolution is mainly driven by the selection parameter. Moreover, based on parameter values, these two calculations of the waiting time to reach cell-size stability are both in line with the results of the long-term experiment.

In summary, it can be said that the analytical and numerical analysis of the waiting times derived from two different mathematic models of cell-size evolution in experimental $E$. coli populations strengthen the claim that the clonal expansion rate of selectively advantageous organisms are the largest driving force.

\section{ACKNOWLEDGMENTS}

Research supported by an NNSFC grant (\#14Z103010221) to B. Yuan.

Genetics and Molecular Research 15 (3): gmr.15038991 


\section{REFERENCES}

Armitage P and Doll R (1954). The age distribution of cancer and a multi-stage theory of carcinogenesis. Br. J. Cancer 8: 1-12. http://dx.doi.org/10.1038/bjc. 1954.1

Barrick JE, Yu DS, Yoon SH, Jeong H, et al. (2009). Genome evolution and adaptation in a long-term experiment with Escherichia coli. Nature 461: 1243-1247. http://dx.doi.org/10.1038/nature08480

Beerenwinkel N, Antal T, Dingli D, Traulsen A, et al. (2007). Genetic progression and the waiting time to cancer. PLoS Comput. Biol. 3: e225.http://dx.doi.org/10.1371/journal.pcbi.0030225

Chen CW (1993). Armitage-Doll two-stage model: implications and extension. Risk Anal. 13: 273-279. http://dx.doi. org/10.1111/j.1539-6924.1993.tb01079.x

Corless RM, Gonnet GH, Hare DEG, Jeffrey DJ, et al. (1996). On the Lambert W Function. Adv. Comput. Math. 5: 329359. http://dx.doi.org/10.1007/BF02124750

Cui FS and Yuan B (2012). Analysis of morphological evolution in a long-term experiment with Escherichia coli. Proc. Int. Conf. Systems Biol. 1: 211-215.

Cui FS and Yuan B (2015). Evolutionary dynamics of morphological stability in a long-term experiment with Escherichia coli. IET Syst. Biol. 9: 25-30.

Durrett R, Schmidt D and Schweinsberg J (2009). A waiting time problem arising from the study of multi-stage carcinogenesis. Ann. Appl. Probab. 19: 676-718. http://dx.doi.org/10.1214/08-AAP559

Elena SF and Lenski RE (2003). Evolution experiments with microorganisms: the dynamics and genetic bases of adaptation. Nat. Rev. Genet. 4: 457-469. http://dx.doi.org/10.1038/nrg1088

Futuyma DJ (2010). Evolutionary constraint and ecological consequences. Evolution 64: 1865-1884. http://dx.doi. org/10.1111/j.1558-5646.2010.00960.x

Gerstung M and Beerenwinkel N (2010). Waiting time models of cancer progression. Math. Popul. Stud. 17: 115-135. http://dx.doi.org/10.1080/08898480.2010.490994

Graña M and Acerenza L (2001). A model combining cell physiology and population genetics to explain Escherichia coli laboratory evolution. BMC Evol. Biol. 1: 12-20.http://dx.doi.org/10.1186/1471-2148-1-12

Heidenreich WF (1996). On the parameters of the clonal expansion model. Radiat. Environ. Biophys. 35: 127-129. http:// dx.doi.org/10.1007/BF02434036

Hoogenveen RT, Clewell HJ, Andersen ME and Slob W (1999). An alternative exact solution of the two-stage clonal growth model of cancer. Risk Anal. 19: 9-14. http://dx.doi.org/10.1111/j.1539-6924.1999.tb00381.x

Huey RB, Gilchrist GW, Carlson ML, Berrigan D, et al. (2000). Rapid evolution of a geographic cline in size in an introduced fly. Science 287: 308-309. http://dx.doi.org/10.1126/science.287.5451.308

Iwasa Y, Michor F and Nowak MA (2004). Stochastic tunnels in evolutionary dynamics. Genetics 166: 1571-1579. http:// dx.doi.org/10.1534/genetics.166.3.1571

Jablonski D (2000). Micro- and macroevolution: scale and hierarchy in evolutionary biology and paleobiology. Paleobiology 26: 15-52.

Knudson AG, Jr. (1971). Mutation and cancer: statistical study of retinoblastoma. Proc. Natl. Acad. Sci. USA 68: 820-823. http://dx.doi.org/10.1073/pnas.68.4.820

Lenski RE (2004). Phenotypic and genomic evolution during a 20,000-generation experiment with the bacterium Escherichia coli. Plant Breed. Rev. 24: 225-265.

Lenski RE and Travisano M (1994). Dynamics of adaptation and diversification: a 10,000-generation experiment with bacterial populations. Proc. Natl. Acad. Sci. USA 91: 6808-6814.http://dx.doi.org/10.1073/pnas.91.15.6808

Lenski RE, Rose MR, Simpson SC and Tadler SC (1991). Long-term experimental evolution in Escherichia coli. I. adaptation and divergence during 2,000 generations. Am. Nat. 138: 1315-1341. http://dx.doi.org/10.1086/285289

Mahler DL, Revell LJ, Glor RE and Losos JB (2010). Ecological opportunity and the rate of morphological evolution in the diversification of Greater Antillean anoles. Evolution 64: 2731-2745. http://dx.doi.org/10.1111/j.1558$\underline{5646.2010 .01026 . \mathrm{x}}$

Márquez-García M, Correa-Solis M, Sallaberry M and Méndez MA (2009). Effects of pond drying on morphological and life-history traits in the anuran Rhinella spinulosa (Anura: Bufonidae). Evol. Ecol. Res. 11: 803-815.

Matamoro-Vidal A, Furness CA, Gouyon PH, Wurdack KJ, et al. (2012). Evolutionary stasis in Euphorbiaceae pollen: selection and constraints. J. Evol. Biol. 25: 1077-1096. http://dx.doi.org/10.1111/j.1420-9101.2012.02494.x

Moolgavkar SH (1978). The multistage theory of carcinogenesis and the age distribution of cancer in man. J. Natl. Cancer Inst. 61: 49-52.

Moolgavkar SH and Venzon D (1979). Two-event models for carcinogenesis: incidence curves for childhood and adult tumors. Math. Biosci. 47: 55-77. http://dx.doi.org/10.1016/0025-5564(79)90005-1

Genetics and Molecular Research 15 (3): gmr.15038991 
Moolgavkar SH and Knudson Jr AG (1981). Mutation and cancer: a model for human carcinogenesis. J. Natl. Cancer Inst. 66: 1037-1052.

Philippe N, Pelosi L, Lenski RE and Schneider D (2009). Evolution of penicillin-binding protein 2 concentration and cell shape during a long-term experiment with Escherichia coli. J. Bacteriol. 191: 909-921. http://dx.doi.org/10.1128/ JB.01419-08

Schöllnberger H, Beerenwinkel N, Hoogenveen R and Vineis P (2010). Cell selection as driving force in lung and colon carcinogenesis. Cancer Res. 70: 6797-6803. http://dx.doi.org/10.1158/0008-5472.CAN-09-4392

Schweinsberg J (2008). The waiting time for m mutations. Electron. J. Probab. 13: 1442-1478. http://dx.doi.org/10.1214/ EJP.v13-540

Stanek MT, Cooper TF and Lenski RE (2009). Identification and dynamics of a beneficial mutation in a long-term evolution experiment with Escherichia coli. BMC Evol. Biol. 9: 302-314.http://dx.doi.org/10.1186/1471-2148-9-302

Thomas CD, Bodsworth EJ, Wilson RJ, Simmons AD, et al. (2001). Ecological and evolutionary processes at expanding range margins. Nature 411: 577-581. http://dx.doi.org/10.1038/35079066

Woods R, Schneider D, Winkworth CL, Riley MA, et al. (2006). Tests of parallel molecular evolution in a longterm experiment with Escherichia coli. Proc. Natl. Acad. Sci. USA 103: 9107-9112. http://dx.doi.org/10.1073/ pnas.0602917103

Genetics and Molecular Research 15 (3): gmr.15038991 